\title{
Pruning Forests to Find the Trees*
}

\author{
Hasan M. Jamil \\ Department of Computer Science \\ University of Idaho \\ jamil@uidaho.edu
}

\begin{abstract}
The vast majority of phylogenetic databases do not support a declarative querying platform using which their contents can be flexibly and conveniently accessed. The template based query interfaces they support do not allow arbitrary speculative queries. While a small number of graph query languages such as XQuery, Cypher and GraphQL exist for computer savvy users, most are too general and complex to be useful for biologists, and too inefficient for large phylogeny querying. In this paper, we discuss a recently introduced visual query language, called $P h y Q L$, that leverages phylogeny specific properties to support essential and powerful constructs for a large class of phylogentic queries. Its deductive reasoner based implementation offers opportunities for a wide range of pruning strategies to speed up processing using query specific optimization and thus making it suitable for large phylogeny querying. A hybrid optimization technique that exploits a set of indices and "graphlet" partitioning is discussed. A "fail soonest" strategy is used to avoid hopeless processing and is shown to produce dividends.
\end{abstract}

\section{CCS Concepts}

-Information systems $\rightarrow$ Query optimization; Query languages for non-relational engines; Query planning; Semi-structured data; Web interfaces; •Human-centered computing $\rightarrow$ Graphical user interfaces; Web-based interaction; Graph drawings; $\bullet$ Applied computing $\rightarrow$ Bioinformatics; Genomics; •Computing methodologies $\rightarrow$ Logic programming and answer set programming;

\section{INTRODUCTION}

The interest in developing a flexible, expressive and efficient structure querying engine for phylogenetic databases has been gaining steady popularity [2, 8, 18, 38, 50, 60, 62].

\footnotetext{
* Research supported in part by National Science Foundation grant DRL 1515550.

Permission to make digital or hard copies of all or part of this work for personal or classroom use is granted without fee provided that copies are not made or distributed for profit or commercial advantage and that copies bear this notice and the full citation on the first page. Copyrights for components of this work owned by others than ACM must be honored. Abstracting with credit is permitted. To copy otherwise, or republish, to post on servers or to redistribute to lists, requires prior specific permission and/or a fee. Request permissions from permissions@ acm.org.

SSDBM '16, July 18-20, 2016, Budapest, Hungary

(C) 2016 ACM. ISBN 978-1-4503-4215-5/16/07 . . \$15.00

DOI: http://dx.doi.org/10.1145/2949689.2949697
}

This interest is based in part on the observations that 1) various types of evolutionary data are being generated using extremely expensive algorithms and for life sciences research $[11,41,43]$ and being stored in public databases $[7,23$, $27,55]^{1}$, and 2) their unique properties were not exploited to develop scalable methods for the storage and manipulations of such vast collections of complex data structures $[10,30,45]$. Although phylogenies are fundamentally trees, it was observed that most well developed data manipulation techniques for graphs and trees are rendered ineffective or have exhibited unacceptable performance in phylogenetic databases. However, recent advances in graph and complex structured data management $[3,12,31,49,61,63]$ simultaneously show promise for a well rounded phylogenetic data management system and raise new research questions that need to be addressed.

While the recent graph matching algorithms are efficient, they are not directly suitable as a query language to support features such as part fixed and part tentative structure matching, or for computing wildcard queries such as least common ancestor or reachable nodes. The handful of languages that support declarative querying, do so incurring a high maintenance and query processing overhead. For example, to reduce the time complexity in LCA (least common ancestor) queries, PhyloFinder [7] preprocesses the trees, and stores additional labeling information in nodes; and Crimson [62] used Dewey node labeling [54]. Although Dewey labeling helps, it often require long nested tree representation. The Crimson system eliminates this problem by storing the labels in nested subtrees to avoid long chains. Such labeling also complicates updates because insertion and modifications disrupt Dewey order, and must now be recomputed. To deal with such labeling hurdles, nested interval encoding [52] was used in PhyloFinder, which translates essentially into a simple string search.

Evidently, the ability to conveniently store phylogenies computed using CPU intensive algorithms [44, 13, 56, 6, $19]$, and later retrieve interesting phylogenies for analyses is increasingly becoming an imperative with the rapid growth in such stored phylogenies. So is the need for a convenient representation for effective integration of various types of phylogenies. From these standpoints, a format and application independent abstract data model, and a declarative query language can play a transformative role [35] in phylogenetic databases. Thus, for declarative phylogeny query

\footnotetext{
${ }^{1}$ Also in many specialized phylogenetic databases such as FUNYBASE [28], DarkHorse [37], TreeFam [25], PALI [47], ImmTree [32], and HvrBase++ [20].
} 
languages, efficient query processing and optimization become a serious next step. The PhyloBase model and the PhyQL query language we present in this paper address both. To our knowledge, PhyQL is one of a handful of languages that allow declarative querying (other than PQL [22] and Crimson), and the only language that allows composable structure and pattern queries visually due to its declarative foundation. In this paper, our focus is on query processing and optimization in PhyQL (introduced recently in [18]) that leverage a deductive reasoner as its query engine.

\section{RELATED RESEARCH}

While a large number of phylogenetic analysis tools and applications have been developed [2, 5, 8, 21, 38, 58, 60], very few query languages are available for phylogenetic databases. Many of these tools are focused on generating phylogenies or using phylogenies already collected in specific formats. Databases such as TreeBASE [55] and PhyloExplorer [38] support custom APIs to search phylogenies in specific formats that are not actually based on tree query languages, and are often based on tree matching algorithms [42]. There has been previous efforts in developing query languages, declarative languages to be specific, for phylogenetic databases $[8,18,22]$ based on the observations in $[30,33]$ with varying degrees of success. The limitations of these languages are still forcing procedural extensions of languages such as Python and Java [9, 15, 36, 48, 50] for accessing and visualizing phylogenies and forcing the user to incur significant development costs.

The PQL language [22], though declarative and can be used to query phylogenies, is specifically designed for querying pathways, have a complex syntax and semantics, and is not amenable to developing a simple and intuitive visual interface. The CDAO-Store on the other hand is designed to support integrated phylogenetic analysis based on NexML format. Although it is based OWL and a logic based model, it only supports a web browser and predefined query suits for accessing its content for specific set of data sources in a limited way. While Nakhleh et. al. [30] had proposed a declarative engine to process tree queries based on a similar canonical model as PhyloBase, its performance was a limiting factor as it used traditional negation based rules to compute LCA queries that forced expensive stable model computation in Datalog.

\section{A MODEL FOR REPRESENTING AND QUERYING PHYLOGENIES}

Over the years, researchers have tried to develop a canonical data model for phylogenies and have proposed several standards for representation. In some ways, all of these models have strengths and deficiencies relative to the applications they aim to support. Consequently, several popular standards have evolved and influenced the analytics researchers use. To reconcile the heterogeneities of these standards and help cross reference data from various models, many mapping and translation methods have also been developed, indicating that these standards are here to stay for an indefinite period. An interesting observation is that deviating from these standards for the purpose of representation, querying and manipulation, and query optimization in no way limits the strengths of any new model since developing a mapping procedure addresses the all too common and prevailing standardization disparity. In other words, developing a generalized data model for the representation of phylogenies for our purposes is academic and follows standard practices.

\subsection{PhyloBase Data Model}

PhyloBase is capable of modeling phylogenies as sets of multi-modal trees with hybridization or horizontal transfers across nodes within a phylogeny. The accommodation of hybridization events basically renders the model to be DAGs as opposed to trees, but treating them orthogonally as exceptions makes them trees nonetheless. The formal model discussed below can be tailored appropriately to accommodate most of the popular standards which treats phylogenies as rooted trees in which internal nodes and edges are possibly labeled, but all leaf nodes are labeled. A set of homogeneous phylogenies (trees) is called collections (or forests), and a database essentially is a set of such collections.

Technically, the language $\mathbb{L}$ of PhyloBase is a structure of the form $\langle\mathcal{I}, \mathcal{V}, \mathcal{L}, \mathcal{C}, \lambda\rangle$ where $\mathcal{I}$ is a set of identifiers, $\mathcal{V}$ is a set of vertices, $\mathcal{L}$ is a set of labels including empty labels, $\mathcal{C}$ is a set of collections, and $\lambda$ is a labeling function. Each phylogeny $T$ in a collection $C$ is of the form $T=\left\langle I, V, E_{a}, E_{h}\right\rangle$ where $I \in \mathcal{I}, V \subseteq \mathcal{V}$ is a set of vertices, and $E_{a} \subseteq V \times V$ and $E_{h} \subseteq V \times V$ are sets of edges such that $\left\langle v_{1}, v_{2}\right\rangle \in E_{a} \Rightarrow\left\langle v_{1}, v_{2}\right\rangle \notin E_{h}$ and vice versa, $E_{a} \cup E_{h}$ is acyclic, $\forall v \in V\left\{\exists u \in V\left\{\langle u, v\rangle \in E_{a} \vee\langle v, u\rangle \in E_{a}\right\}\right\}$ (i.e., $|V| \geq 2)$, and $E_{a}$ is a tree. Since $E_{h}$ models horizontal transfers, we impose the constraint that $\forall v_{1}, v_{2}\left\{\left\langle v_{1}, v_{2}\right\rangle \in E_{h}\right.$, neither $v_{1}$ nor $v_{2}$ is the root node in $\left.T^{2}\right\}$. The set of all such collections $C$ is the set $\mathcal{C}$, i.e., $\mathcal{C}=\bigcup C$.

We require $\lambda$ to be a labeling function of the form $\lambda: U \rightarrow$ $L \times L \times \ldots \times L$ that assigns an n-ary vector of labels where $U$ is one of the components in $\left\{I, V, E_{a}, E_{h}\right\}$. Intuitively, this means every tree $T \in C$ and $C \in \mathcal{C}$ is unique (identified by its ID $I$ ), and is possibly described using attributes (i.e., $\lambda: I \rightarrow L \times L \times \ldots \times L)$, such as author and date. Each edge in $E_{a}$ and $E_{h}$ may also be optionally labeled. Finally, although we allow labeling of any node in $V$, we require that all leaf nodes $v \in T$ to be labeled, i.e., $\forall v, v \in V(\langle u, v\rangle \in$ $\left.E_{a} \wedge \nexists w, w \in V\left(\langle v, w\rangle \in E_{a} \Rightarrow \lambda(v) \neq \emptyset\right)\right)$. This definition allows a subset of internal nodes and edges to be labeled while the rest are possibly not. Finally, we adhere to and use standard terms of graphs and trees such as height or depth of trees, path between nodes, nodes, branching factor or fan out, and average fan out of a node, internal and leaf nodes, and subtrees.

\subsection{Persistent Storage Model}

Given that phylogenies are potentially more than a million level deep, often have several millions of species and many millions of internal nodes, literally storing them as trees is both infeasible and not prudent from management and querying standpoints. The sheer size of the real life phylogenies also challenges the wisdom of the algorithms that try to match them in memory [4] using the tree at a time paradigm. Furthermore, Felsenstein [10] estimated that there are about $8.8 \times 10^{23}$ possible tree topologies just

\footnotetext{
${ }^{2}$ Consider a tree with edges $E_{a}=\{\langle a, b\rangle,\langle a, c\rangle,\langle c, d\rangle\}$. While horizontal transfers captured by $E_{h}=\{\langle b, c\rangle,\langle b, d\rangle\}$ are possible, the set $E_{h}=\{\langle b, c\rangle,\langle d, b\rangle\}$ is not possible because $E_{a} \cup E_{h}$ will now include a cycle via $\langle c, d\rangle$ back to $b$, which is disallowed.
} 
for a 20 species phylogeny, and taxonomists must account for these large number of trees in their analysis. That also means many biologists will hypothesize a significant subset of these theories and would like to store them until a consensus is reached. In TreeBASE [55] alone, there are about 15,000 stored phylogenies as of 2010 . So, it is imperative that we develop a storage model that helps efficient search and retrieval through the trees in order to isolate and find the tree of interest or part of it from a large collection.

One simple model is to store each edge of a tree as a binary pair, possibly indexed with a tree identifier to recognize its membership in a tree. The major cost incurred in this model, however, is in assembling the trees from the component edges to match the query tree topology. Nonetheless, this simple model has been the main choice so far for many phylogenetic database systems including TreeBASE/ TreeSearch [30, 42, 55], Crimson [62], CDAO-Store [8], PhyQL [18] and PhyloFinder [7]. Early research in [30, 42] contrast the advantages and disadvantages of this simple flat edge based representation with respect to a query engine called TreeSearch that supports various phylogeny querying features not available in traditional tree searching systems. One of the complex query features of LCA computation for arbitrary number of labeled or unlabeled tree nodes have been shown to be particularly expensive. In this paper, we examine the possibility of using a more complex representation as a storage model and show that such a model holds promise and delivers significant performance tradeoffs.

\subsubsection{Hub and Spoke versus Edge Representation}

The encouraging development lately is that research in nested structures such as XML has made significant advances in terms of indexing, storage and retrieval, especially for very shallow structures [1, 29]. A recent graph matching technique [39, 40, 59] also used complex XML structures as a storage and matching unit called graphlet, demonstrating the power this approach holds. These research are our inspiration to use a more complex structure, a node neighborhood - a node and all its descendants - as the smallest unit of representation and storage, with the hope to have more acceptable retrieval efficiency, as opposed to storing a whole tree as an XML document, or just the edges as in relational models. The added advantage of using such a moderately complex structure as a unit allows for including some structural cues into the representation and leverages the constraints imposed by them. It also helps retrieve a target structure of choice as a building block toward assembling a tree as opposed to using only edges that requires substantial assembling efforts.

To illustrate the advantage of the hub and spoke representation we adopt in PhyloBase, consider the partially labeled hypothetical Myosin gene evolution tree $t_{1}$ adapted from [46] in figure 1(a), showing a hybridization event as the red edge. As opposed to storing all the edges, we store the three hubs and the hybridization edge as shown in figure 1(b) covering all the edges. Note that we also match and retrieve hubs, not edges, as a single and minimum unit. That means, to reconstruct the tree, we assemble these hubs judiciously, i.e., join them in proper order, in ways similar to lego blocks. For example, to compute the query $q_{1}$ in figure $1(\mathrm{c})$, we search all the hubs in a collection to retrieve hubs $h_{1}$ with a leaf node labeled myh4 and and edge labeled 0.262, and $h_{3}$ with one leaf node labeled $m y h \%$, and then assemble to match the

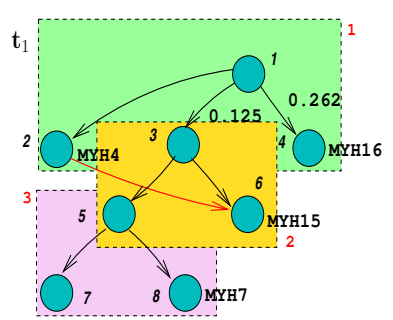

(a) A hypothetical phylogeny with three hubs.

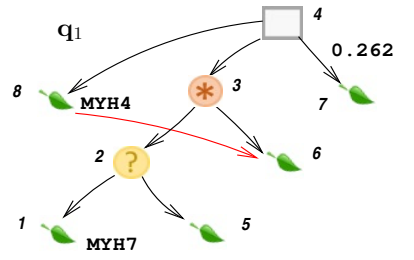

(c) An example PhyQL query (d) Modified query $q_{2}$ of $q_{1}$ using visual icons.

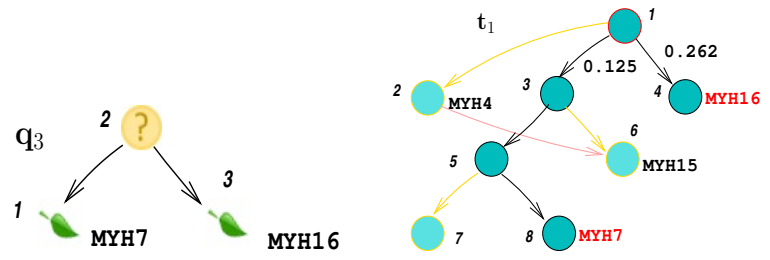

(e) A PhyQL LCA query. (f) Highlighted $t_{1}$ matching LCA query $q_{2}$ in figure $1(\mathrm{e})$.

Figure 1: PhyQL tree representation: (a) decomposition for storage, and (b) on demand reassembling.

structure and semantics ${ }^{3}$ of the query $q_{1}$, and return the entire tree $t_{1}$ in figure 1 (a). We will do so by finding the hub $h_{2}$ that is a descendant of node 1 in hub $h_{1}$ and has a leaf node that is connected with the leaf node in hub $h_{1}$ labeled myh4 through a hybridization event. Note that modifying query $q_{1}$ to $q_{2}$ will generate no (empty) response - we can match everything but query node 4 , which is an internal node, not a root node. Similarly, in response to query $q_{3}$ in figure 1(e), which basically asks for all the trees with the least common ancestor of myh7 and myh16, PhyQL will return the subtree in figure $1(\mathrm{f})$ as highlighted ${ }^{4}$.

\subsubsection{Hub Storage as XML Documents}

We adapt the concept of graphlets $[57,16]$ to represent phylogenetic trees as a set of decomposed structures we call $h u b s$, where we model every internal node (including the root) as a hub to which all its descendants are connected. A tree is thus a set of hubs and a set of hybridization events modeled as a set of edges. A tree in PhyloBase therefore is an XML document at most five levels deep as shown in figure 2 , which captures the tree $t_{1}$ in figure 1(a) entirely.

In this XML representation, the tags are not necessarily

\footnotetext{
${ }^{3} \mathrm{~A}$ brief overview of the PhyQL visual query language is presented in section 3.3 .

${ }^{4}$ On tree $t_{2}$ in figure $4(\mathrm{a})$, on the other hand, this query will return the subtree under node 7 .
} 


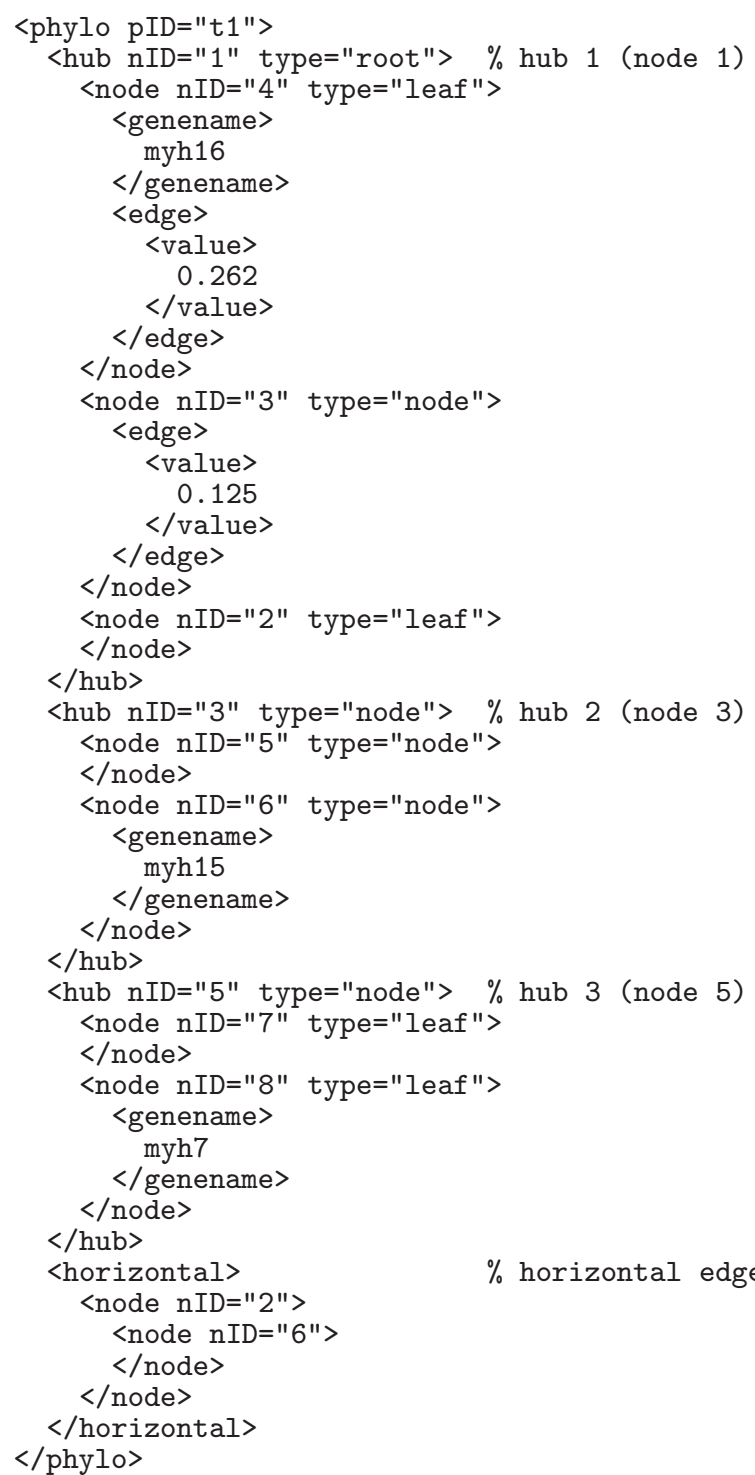

Figure 2: XML representation of tree $t_{1}$.

standard, but what is customary is that the top element is a

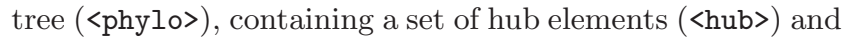
hybridization elements (<horizontal>). Each hub element consists of a set of edges, along with edge labels (<edge >) and child label (<genename $>$ ). The tag names depend on the application scheme for the trees, and are unimportant. The extra element <value> in <edge> allows for multiple attributes for an edge which is distinct from the node labels. If more than a single label is needed for a node, they can be listed in an analogous manner to the tag <genename>. Hybridization edges can be labeled simply and identically to the node labels as a list of elements under the first <node> element in the <horizontal> set.

\subsection{PhyQL Visual Query Language}

Once stored, phylogenies in PhyloBase can be retrieved and manipulated using an user interface that allows writing queries using visual icons, and supports powerful operations in ways similar to SQL in relational databases, and XQuery in XML databases. In this section, we briefly introduce the PhyQL visual query language and its interface. Note that the language is not the focus of this paper, its query optimization strategies are. The operations and classes of queries supported in PhyQL are Lookup or selection, Shred or projection, Graft or join, and Match or top- $k$ tree matching. These operations map collections to collections yielding a closed language making it possible to support complex nested queries. Except for join and match, which are binary, all operations are unary. Since we are interested mainly in query optimization issues, for the purpose of this paper, we will only discuss the Lookup operation and defer the discussion on the remaining query types and other interface features to another article.

The PhyQL user interface in figure 3 consists of five subsystems - Visual Editor, Syntax Analyzer, Visualizer and Browser, Buffer Manager, and Import and I/O Unit. Users use the editor to construct queries using the eight visual icons shown on the vertical panel under the Query tab on the left by drawing query phylogenies on the canvas. Query responses are returned as a clickable list on the right lower corner frame. The editor canvas splits into two frames to display returned responses once one of the links is clicked for visualization and exploration in one of four supported layouts, i.e., cladogram, hierarchical, phylogram and tree. The response trees are stored in a system buffer and can be used for secondary querying individually or as a collection. The query tab also shows all the supported operations as selectable buttons on the top horizontal panel. For auxiliary database management operations, a set of tabs is available. The editor is implemented as an open source web accessible query front-end using Java and mxGraph graph library.

\subsection{PhyQL Syntax and Semantics}

The syntax of PhyQL supports three icons for three types of nodes: Root (a white square), (internal) Node (a gray circle), and Leaf (a green leaf); three wildcard icons to support query flexibility: Any (a starred pink circle), LCA (a question mark inside a mustard circle) and Subtree (a pink and blue tree); and two edge icons to capture node relationships: Edge (blue edge) and HEdge (red edge). These icons have predefined meanings and are implemented as first-order predicates. Users construct a query by judiciously assembling them in a tree that follows the PhyQL construction rules or grammar, and is semantically meaningful. The construction process is interactive and thus syntactic errors are detected in real time. Users are able to choose an icon, and drop it on the canvas. The selection remains active until another icon is chosen or a query operation is performed. The icons can be instantiated or labeled with constants using the context sensitive form active for the selected icon on the upper right corner. The entries on the form depend on the scheme of the phylogeny collection, and the types chosen for each of the entries.

The semantics of the icons are simple and intuitive. A Root icon represents a node for which there is no parent. An internal Node has at least two children and possibly a parent, whereas a leaf node will never have a child and always have a single parent. All nodes are connected to other nodes using Edge icons. Some nodes are connected via the HEdge icons. Since hybridizations are lateral edges between nodes, and are not parent-child relationships, a node usually 


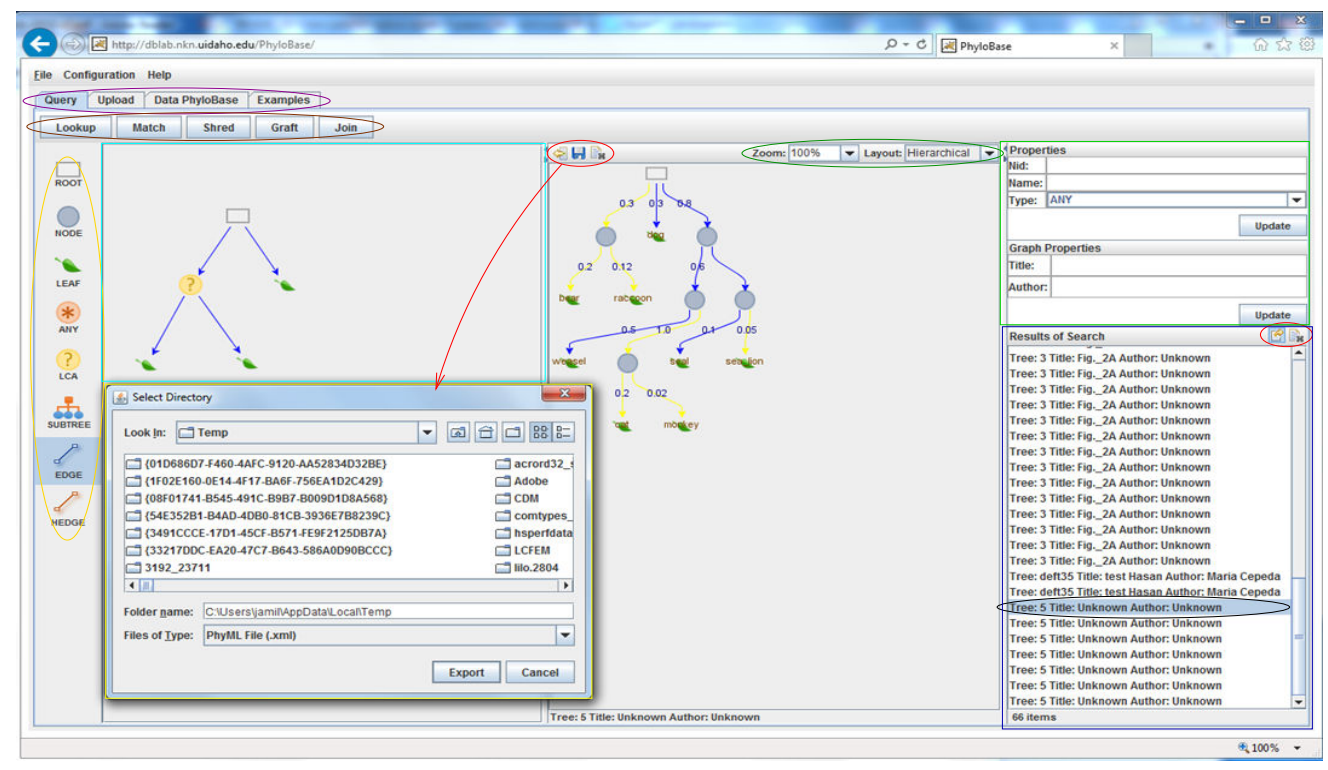

Figure 3: PhyloBase user query interface for PhyQL.

has one such edge as shown in figure 1(c) (between nodes 8 and 6). Among the tree wildcard icons, only $L C A$ icon is required to have at least two children, and may not have a parent. In contrast, Any and Subtree must have at most one parent, and Subtree cannot have any children. The wildcard icons mainly support arbitrary structure computation that cannot be fixed ahead such as reachability or paths, LCA of a set of nodes, or an arbitrary subtree. Since these are fundamentally computational, they are implemented as deductive rules, as opposed to base predicates. The next few examples clarify the semantics of these icons.

Consider the query $q_{1}$ over the phylogeny $t_{1}$ in figure 1 again. As mentioned before, this query returns the entire tree $t_{1}$. This is because the LCA of myht and another leaf node is node 2 in query $q_{1}$. Similarly, query node Any in node 3 requires at least one node to be between query nodes 4 and 2 which is parent to a leaf node. Finally, we require node 8 labeled with myh4 to be connected to node 6 via hybridization. We can match this pattern along with all the constraints with the tree $t_{1}$. However, this pattern cannot be matched with tree $t_{2}$ in figure 4 (a) because while query node 2 can be matched with node 7 in $t_{2}$ being the least common ancestor of nodes 13 and 9 , we cannot match query node 4 to node 2 in $t_{2}$ since it is not a root node. But if we replace node 4 with an internal node in the query as in query $q_{2}$ in figure $1(\mathrm{~d})$, we will be able to match. Furthermore, if we also remove the hybridization event from node 8 to node 6 , we can have two solutions: by mapping query nodes 2 and 3 respectively to data node pairs 7 and 5 , and 10 and 7 .

\section{PROLOG TREE META-INTERPRETER}

The basic implementation strategy used in PhyloBase is to use query translation to transform PhyQL queries into an equivalent Datalog subgraph isomorph matching query, and use a reasoning engine such as Prolog to respond to queries. An alternative choice would be to use a subgraph matching algorithm directly, but that essentially makes PhyQL wild- card queries harder to implement. Wildcard queries are very powerful in phylogenetic databases and add significant flexibility to the system that many similar databases lack [42, 30, 62]. For example, TreeBASE, Tree of Life and iTOL only support fixed structure and template based queries, and thus do not help users retrieve tentative target trees in flexible ways. In these databases, users are forced to speculate the structure and find the target trees by trial and error, which potentially is a blind and very long process.

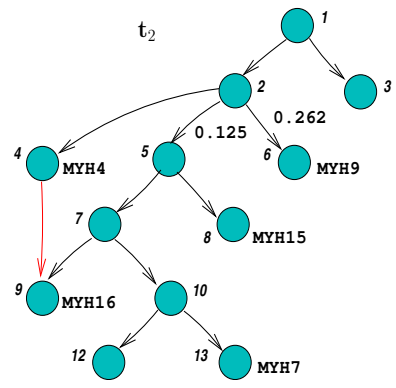

(a) Example phylogeny $t_{2}$.

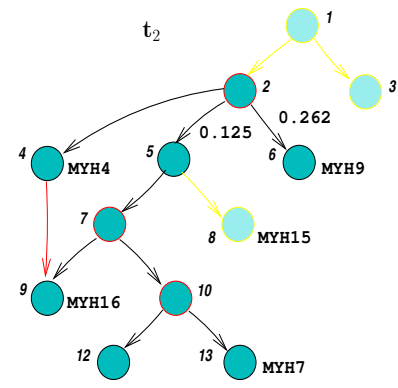

(b) Showing part of $t_{2}$ matching query $q_{2}$ in figure $1(\mathrm{~d})$.

Figure 4: PhyQL query semantics.

The process of translation to Prolog is intuitive and simple. For example, the query in figure 1(c) can be expressed in Prolog as follows. Recall that we do not store every node or edge individually, instead we store and manage hubs. Thus inspecting a child node stored as part of a hub requires consulting the entire hub. The hub representation closely follows the structures in figures 1(b), 2 and $7(\mathrm{a})$ and is cast as the predicate called $h u b$ as follows.

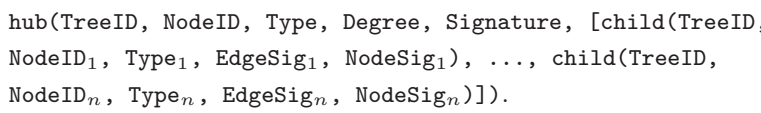


The Prolog representation for the three hubs in figure 1(a) accordingly is shown below.

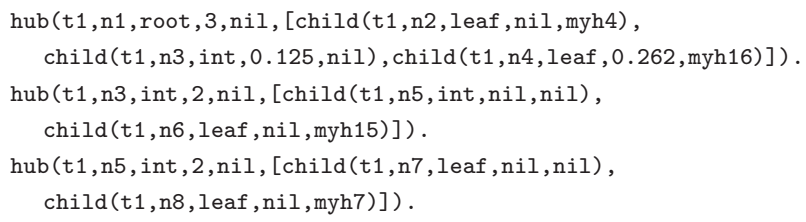

Finally, we represent the hybridization edges with the predicate hedge as follows for the edge between nodes 2 and 6 .

hedge (t1, n2, n6, nil).

The PhyQL query in figure 1(c) is translated into the following Prolog conjunctive query using a simple algorithm discussed briefly ${ }^{5}$ in section 6 . This query actually identifies the response in figure 1(a), and fails on tree $t_{2}$ in figure 4(a).

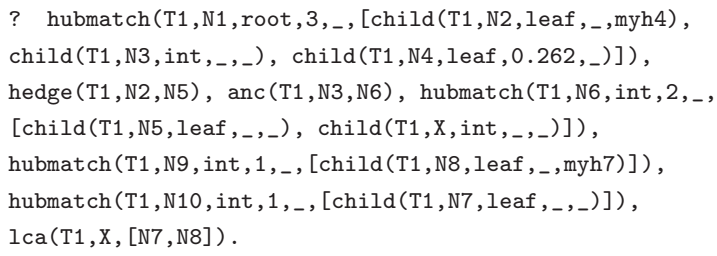

Before we discuss the semantics of these predicates and how they capture PhyQL query semantics, let us introduce the axioms that we use as the front end Prolog interpreter or PhyQL query engine. The best aspect of this engine is that by changing these axioms, and the PhyQL to Prolog translation rules, we can change the semantics of the queries to customize application needs. Since the entire query engine is just these few lines of rules, the overhead is negligible.

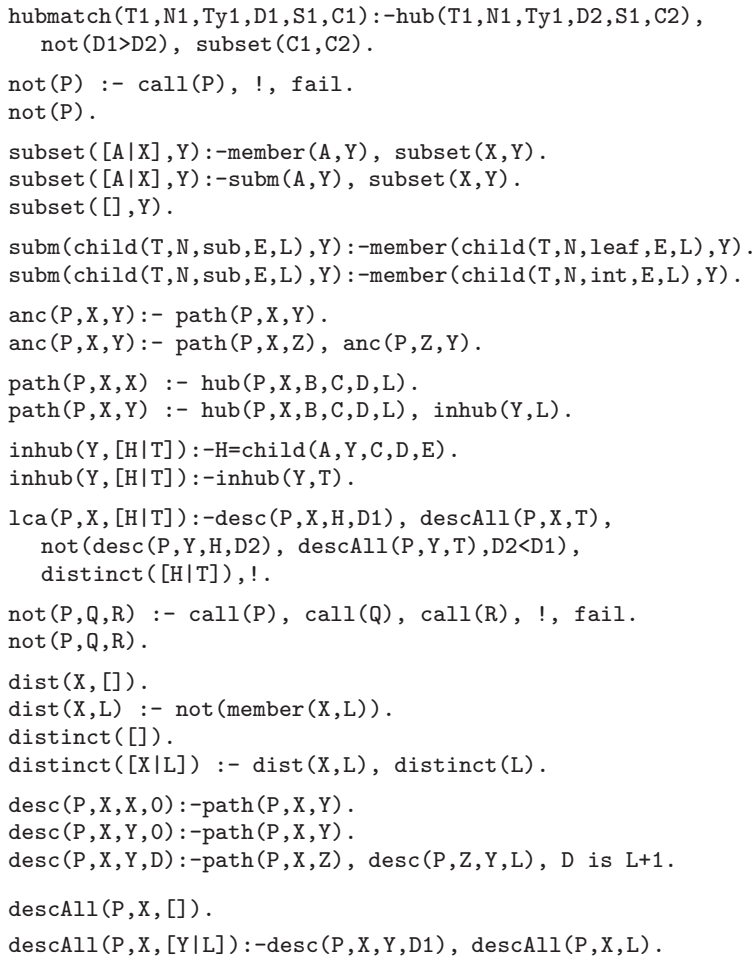

${ }^{5}$ We omit the details of the translation algorithm for brevity largely because query optimization is the focus of this paper.
Although the meaning of these axioms should be immediate, we briefly explain their semantics and discuss how they help processing PhyQL queries. Apart from the two EDB predicates hub and hedge, there are three main IDB rules hubmatch $/ 6$, anc $/ 3$, and $1 \mathrm{ca} / 3$, where $p / k$ means predicate of arity $k$. The other rules (subset $/ 2$, not $/ 1$, not $/ 3$, dist $/ 2$, distinct $/ 1, \operatorname{desc} / 4, \operatorname{descAll} / 3, \operatorname{path} / 3$ and inhub/2) are auxiliary predicates needed for the implementation of the former three, and should be obvious from their context. The trick in the query translation is the proper sequencing of the building blocks using these axioms, first to implement the proper matching of the target tree, and then to ensure an efficient evaluation. We only highlight the first three below.

\section{1 hubmatch Axiom}

Recall that our goal is to find phylogenies in the database for which the query phylogeny is a subgraph isomorph as a structure, but the labels are matched one to one. That means each query phylogeny hub must be a subgraph isomorph, or a substructure, of some data graph. It does so by choosing a data hub which has, at the least, identical number of children and match all the labels - i.e., Ty1, and $\mathrm{S} 1$ are identical in the antecedent and consequent (type and signature of the hubs match), D1 $\leq$ D2 (query hub has no more children than the data hub), and $\mathrm{C} 1 \subseteq \mathrm{C} 2$ (all the children in the query hub are present in the data hub, and their type and signatures match).

One subtlety we must mention here is that we do not have a separate rule to match the wildcard Subtree. Instead we model it as a special case of the hubmatch axiom since a subtree operator is never a hub node, i.e., it will always appear as a leaf node in a PhyQL query. Since a subtree can match with either an internal node or a leaf node, we add the subm rules to match a subtree type with either an internal node or a leaf node separately.

\section{2 anc Axiom}

The anc axiom implements PhyQL's Any operator as a reflexive transitive closure of the path predicate to allow for a node to be its own descendant. We allow reflexivity by observing that the Any operator will also never be a root node in a PhyQL query, but will always be an internal or a leaf node, and is the only operator that allows a single child in a query tree (see node 7 in figure 6 ). We thus include the first and possibly the only node the Any operator must match as part of the hub it belongs to. The path facts are collected for each hub, and its descendants, and the transitive closure is computed using the simple rule as shown.

\section{3 lca Axiom}

Finally, the axiom lca returns the least common ancestor $\mathrm{X}$ of a set of nodes in a phylogeny. The functionality of the LCA rules can be explained using the tree in figure 5. Consider computing LCA of nodes $d$ and $e$ as the evaluation of the goal $l c a(T, X,[d, e])$, which means returning LCA $X$ in tree $T$ for the set of nodes $\{d, e\}$. Since the desc rule computes the transitive closure from leaf to root, the first solution generated will be $X=b$. However, this solution is not accepted until it can be proven that there does not exist another node $X \neq b$ such that the depth of $X$ is smaller than solution $X=b$ (shown as the green solution in figure 5 ). If we consider computing the LCA for $\{j, p, m\}, X=g$ will be the solution for which the depth is minimum. The process 
is shown in successive steps in purple in figure 5 . The cut operator in rule 12 ensures no further exploration once one solution is found, which is always the least solution.

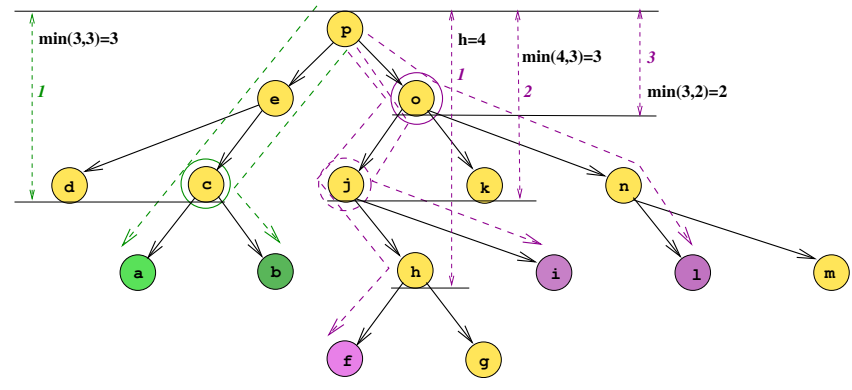

Figure 5: Counting based LCA computation.

\section{PRUNING AIDS}

Careful analysis of the query in figure 1(c) reveals that we are interested in three hubs such that the hub $\mathrm{N} 1$ is a root node with at least three children: one is a leaf node labeled myh4 and another is a leaf node with an edge labeled 0.262. The third child, however, may be a direct descendant, or one far below such that it has two children: one is a leaf node, and another is an internal node, a least common ancestor of two leaf nodes of which one is labeled myh\%. This simple semantics is actually deceptive due to the presence of the two wildcard operators that require intricate handling. Before we discuss how to handle wildcards, let us first review some features all phylogenies share which act as our guide in our query evaluation strategy.

\subsection{Properties of Phylogenies}

In PhyloBase, we observe several distinctive properties of phylogenies that are useful for the design of a phylogenetic query language and a query processor for it. First, although a phylogeny can be considered a graph, it is fundamentally a tree, and so it consists of parent-child relationships (as well as rare horizontal events), and more importantly, is not a DAG (directed acyclic graph). This observation helped us in proposing a hub and spoke representation for the storage structure as a unit of representation. As such, a leaf node which does not have a child of its own, does not have a structure. Instead, it belongs to a structure of its parent, and thus also belongs to only one structure.

This observation has serious optimization related implications. For example, if a query node has a node or an edge label, and a database hub node does not have either of these labels as a whole, the hub can be eliminated from any matching consideration even if it satisfies other query constraints such as other edge or node label constraints this hub is not a match candidate. Not only this, the entire phylogeny in the database can now be eliminated. Without having the hub and spoke representation, we could not have considered the structural constraint so easily. Although the hub representation and its matching with a query hub as a super structure is tested at run time using the hubmatch rule, we are able to test only viable hubs in most likely trees as discussed in section 5.2.3. Note that a query hub must be a sub-structure of a database hub to be considered a match
- a database phylogeny is a valid response only if the query graph is a subgraph isomorph of the database phylogeny (a graph), and matches all the label constraints. The computational speed up is facilitated in two steps: 1) by picking only the right phylogenies that have the required number of hubs matching all the constraints using the indices discussed in section 5.2 .2 , and 2 ) by sequencing them properly to match the query tree structure using the reasoner.

Second, hybridization events in phylogenies are relatively rare. Thus, treating them not as just another edge, and instead identifying them as special edges, we facilitate pruning whole phylogenies. Phylogenies that do not have the required number of hybridizations with the label constraints in the query need not be considered. The Hybridization index in section 5.2.2 helps achieve this goal.

Finally, the assignment of unique IDs to all nodes in PhyloBase helps compute the wildcard subgoals such as Any and $L C A$ much faster. Ordinarily, these two subgoals are the most expensive ones to compute as they potentially compute large chains of path queries with specific query constraints. These are expensive especially when the query subtree below any of these operators have only a few or no label constraints, more wildcard operators, or more label free nodes and edges. In such cases, node IDs act as the only filter. Once a hub is selected and matched at run time, these unique IDs help select unique hubs to be joined.

Whenever labels, especially node labels are available, they help bind the nodes in Any or LCA subgoals, and these wildcards reduce to all or partially bound "lookup" type evaluations, substantially improving performance. Thus, delaying the computation of these subgoals until all of their nodes get bound is a prudent evaluation strategy.

Several other properties that are unique to phylogenies make it possible to develop efficient indexing schemes to identify and retrieve hubs for the assembling of phylogenies. This is a significant departure from many traditional approache, including complex labeling schemes such as Dewey adopted in systems like Crimson. These schemes require expensive reorganization upon updates without yielding significant overall retrieval efficiency. In databases where trees are frequently curated such as the Tree of Life Web Project [27], such artificial labeling schemes for the sake of access efficiency become a huge impediment. As shown in figure 7(b), by assigning unique IDs to each phylogeny, label and node throughout the database, we are able to create efficient hash indices to locate hubs and nodes in a phylogeny. Apart from the hash indices (not shown explicitly), we have four additional purpose built indices presented next.

\subsection{Access Aids and Meta-Data}

Our goal is to store each hub in a tree along with its signatures sequentially in a contiguous space so that once we are processing a tree, we are not required to access multiple disk spaces to collect all the pieces. Obviously, other physical storage models are also possible. But keeping in mind that we plan to use a top-down Prolog engine, which basically will rely on random access to stored predicates, our model is likely to produce significant performance advantages compared to a naive sequential search used for SLD resolution in traditional Prolog engines. The motivation to use a customized search engine for Prolog stems from the need to store trees as hubs in persistent storage and from the following related observations. 
For a $7200 \mathrm{rpm}$ disk drive with $5 \mathrm{~ms}$ average seek time $T_{s}$, and $0.05 \mathrm{~ms}$ block transfer time $T_{b}$, the time taken (seek time + rotational latency + block transfer time) to randomly read a block is about $9.2 \mathrm{~ms}$ accounting for the $4.1 \mathrm{~ms}$ rotational latency $T_{r}$. Depending on the average size of each hub, we can now calculate the relative estimated time needed to read a set of hubs. If we assume that the hubs are stored contiguously in a file using tree IDs as the primary order and the node IDs as the secondary order, we can read an entire tree using only one seek and one rotational latency. If we also assume that each hub is less than a block size $(k=1)$, or too large to fit in one block and thus spans across multiple blocks $(k>1)$, the effective time $T_{e}$ to read a block is equal to $T_{s}+T_{r}+T_{b} \times k$. The decision to read the hubs in a list $L_{h}$ using a hash index on node IDs will depend on if $T_{\text {ran }}=\left(\left(T_{s}+T_{r}+T_{b} \times k\right) \times\left|L_{h}\right|\right) \leq T_{\text {seq }}=\left(\left(T_{s}+T_{r}\right)+T_{b} \times k \times\right.$ $\left.\left|L_{h}\right|\right)$. If the test is positive, we can use a hash index to fetch the hubs individually to process during subgoal evaluation. Otherwise we can read the hubs sequentially for the tree at hand, using only one seek and rotational latency. The total time needed to process all the hubs in all the trees in a list $L_{t}$ is thus $T_{\text {ran }} \times\left|L_{t}\right|$ or $T_{\text {seq }} \times\left|L_{t}\right|$.

The estimate above is deceptive and not reflective of the fact that queries only express part of a tree and we do not need to fetch the entire tree to respond to one. In reality, some trees need not be considered at all, and for some, matching can be expedited by judiciously ordering the query subgoals. However, there are cases when the matching would fail due to the fact that we are unable to assemble the pieces and we could not predict imminent failure for lack of information. This lack of information is primarily an absence of hub constraints that help predict not only the choice of the hubs in a tree, but also the connectivity between the hubs. This is particularly true for the two wildcard operations of PhyQL, the Any and $L C A$ operators. Let us illustrate this using the example query in figure 6 .

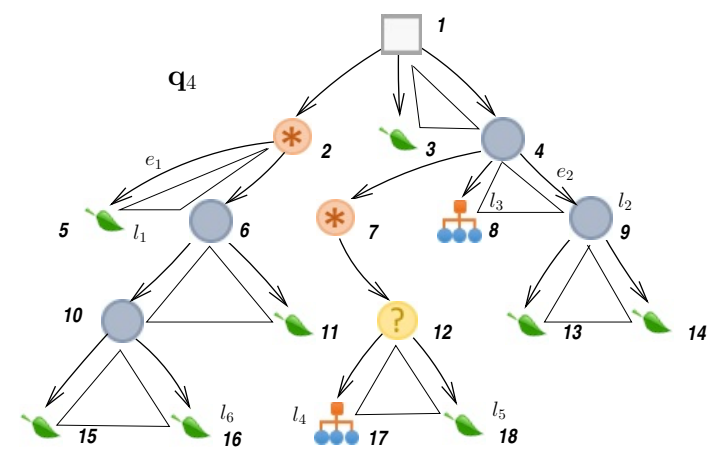

Figure 6: Selectivity based subgoal ordering.

\subsubsection{Cost Estimation of Wildcard Queries}

In query $q_{4}$, there are six fixed structured hubs that we must assemble that are easy to identify: the hubs corresponding to nodes 1, 2, 4, 6, 9 and 10. Although the hub corresponding to node 2 is transitively connected to hub 1 , the length of which we cannot predetermine, we still can preselect hub 2 using the labels of node 5 , and test if a path between the selected node 2 and node 1 exists. The same is not true, however, for the hubs corresponding to nodes 7 , or 12. This is mostly because there are no constraints such as node or edge labels that can be used to uniquely locate nodes in a tree and then test respectively for the transitivity or LCA satisfaction. Only constraint we have is that node 12 must be the LCA of nodes 17 and 18, which are identifiable using their labels. For these nodes belonging to some hub nodes, we can compute the LCA node at run time.

Once we have established node 12, which is always unique, we can fix node 7 (to which node 12 must belong) and then test the transitive connectivity between nodes 7 and 4 , resulting in the most computation intensive and expensive part of this query. However, the Prolog query for $q_{4}$ can be written as follows using the predicates we have introduced in section 4 . In this query, the superscript $f f f$ means the hub node and its first two children have no edge or label constraints, and $f b f$ on the other hand, means the first child has either an edge or a label constraint, and so on. Note that the variable T1 (the tree ID) is always free.

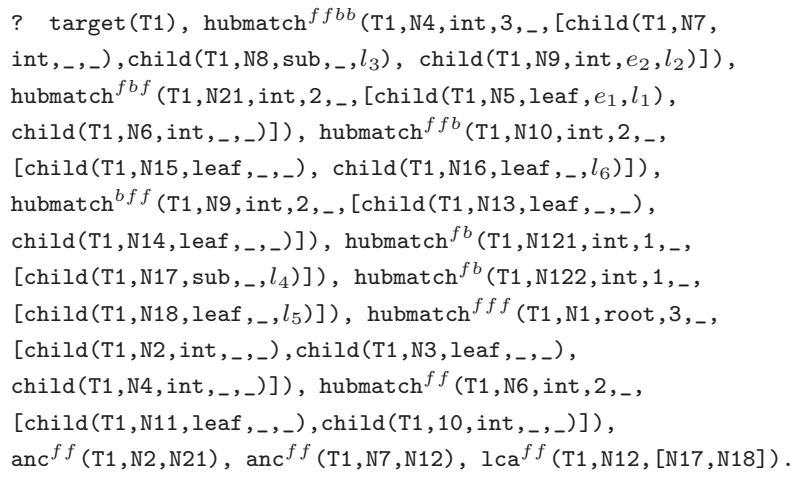

\subsubsection{Hub and Edge Indexing}

Since we store hubs as units, we also index hubs in several ways to allow multiple key access, and to support cost estimation. The keys on which we create indices are node IDs $(N)$, tree IDs $(T)$, hub density $(H)$, node labels $(L)$, edge labels $(E)$, and hybridization events $(B)$. The corresponding indices respectively are Node Description shown in figure 7(a), and Tree Hubs, Hub Density, Node Label, Edge Label and Hybridization as shown in figure 7(b). Each of these indices allows access to the set of hubs matching the search keys to find out how many matching hubs exist. Depending on the key, the index points to an ordered list of hubs $n_{i}$ (as in Tree Hubs), tree and hub ID pairs $\left\langle p_{i}, n_{i}\right\rangle$ (as in Hub Density, Node and Edge Label indices), or pairs of pairs $\left[<p_{i}, n_{i}>,<p_{i}, n_{j}>\right.$ ] (as in Hybridization).

For example, in the Tree Hubs index in figure 7(b), tree 2 consists of 7 hubs including hubs 11 and 17. In this index, all the hub node IDs are kept in an ordered list, and using a hash, the hubs can be retrieved from the index Node Description. Note that the child node IDs are mapped to the parent hubs in Node Description index. The Hub Density index on the other hand serves two purposes. First, for the purpose of estimating how many hubs in the database have 3 or more children, we can use the information in the second column of this index. The cumulative Count column indicates that there are 21 such hubs in the database including hubs $\langle 1,2\rangle,\langle 1,12\rangle,\langle 1,18\rangle$, and $\langle 3,14\rangle$. The list also implicitly includes $\langle 4,26\rangle,\langle 4,51\rangle,\langle 5,7\rangle,\langle 8,61\rangle$ for degree 4 ; $\langle 1,27\rangle,\langle 2,17\rangle,\langle 2,71\rangle,\langle 37,9\rangle$ for degree 5 ; and $<10,1>,<12,107>,<12,111>$ for degree 6 ; and so on, i.e., the higher density hubs in this index, 21 in total. 


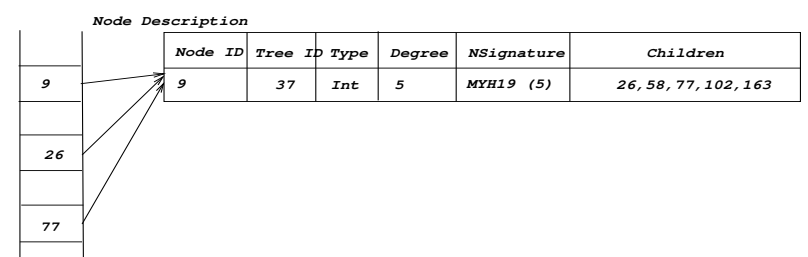

(a) Hub and spoke description of a phylogeny node.
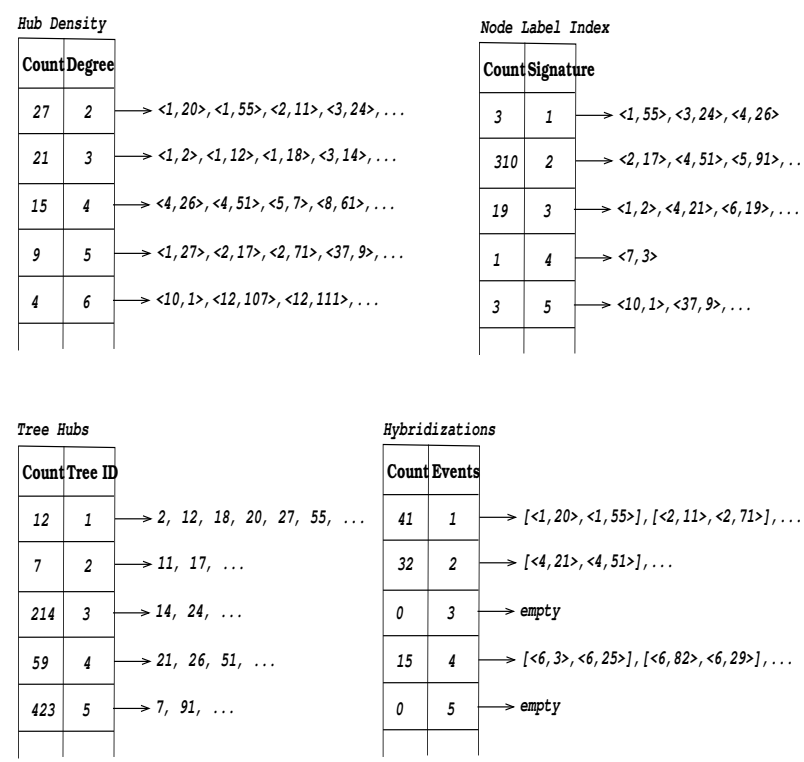

(b) Inverted list indices.

Figure 7: Hub representation and inverted lists.

The Node Label, as well as Edge Label, index points to all nodes in the entire database for a given label. In fact, a label can be composite involving many attributes ${ }^{6}$ we call signature. In this index we use an identifier to represent such a signature. Thus, the fifth entry $(3,5)$ captures the fact that the signature myh19 (represented as its hash value 5) can be found in three trees in the database, and the members $<10,1>$ and $<37,9>$ the index points to mean that hubs 1 and 9 in trees 10 and 37 respectively, all have the signature myh19. Note that according to the phylogeny properties discussed in section 5.1, once we have $\langle 10,1\rangle$ we cannot have other entries in a list that involve tree 10 for myh19.

Finally, Hybridization index lists nodes connected in a tree via hybridization. These are rare events and thus are used as high selectivity predictors. The first row with the entry $(41,1)$ means that there are 41 trees in the database such that each has one hybridization event. The list $[<1,20\rangle$, $<1,55>],[<2,11>,<2,71>]$ that follows the entry, captures the fact that nodes 20 and 55 in tree 1 are connected via hybridization, and so are 11 and 71 in tree 2 . Similarly, row 4 says that there are 15 trees with 4 hybridizations each.

\subsubsection{Estimating the Most Likely Set of Trees}

The Prolog query $q_{4}$ in section 5.2 can be made to execute efficiently in many ways including fail as early as pos-

\footnotetext{
${ }^{6}$ Although, for simplicity and ease of presentation, we only use singular labels in this paper.
}

sible strategy. The indices discussed above can be used to estimate the relative cost of predicate evaluations using a top-down reasoner such as Prolog. If $k_{1} \geq k_{2} \geq k_{3}$ are selectivity estimates ${ }^{7}$ of predicates $p_{1}, p_{2}$ and $p_{3}$ respectively, the order of evaluation $p_{1}, p_{2}, p_{3}$ is likely more efficient than $p_{3}, p_{2}, p_{1}$ if $p_{1}$ or $p_{2}$ fails more often than $p_{3}$. Our goal is to estimate the relative size of the candidate EDB (extensional database) predicates for each of the subgoals and determine an order of evaluation. We design a polymorphic estimator function $\mathcal{F}_{I}(S)$ for each of the indices such that it will return the list it logically points to for signature $S$. The selectivity of a hub $h_{n}$ is the intersection of the functions $\left|\mathcal{F}_{H}\left(S_{h}\right) \cap \mathcal{F}_{L}\left(S_{l}\right) \cap \mathcal{F}_{E}\left(S_{h}\right)\right|$ corresponding to bound hub density, node label and edge label signatures, and the selectivity of hybridization event predicates $e_{m n}$ is $\left|\mathcal{F}_{B}\left(S_{b}\right)\right|$. Since not all trees will satisfy all the predicates matching the target hubs, we design another function $\mathcal{T}$ which returns only the tree IDs that are common in all the lists, and store them in a database predicate target $/ 1$. We add target $/ 1$ as the first subgoal in every query to force evaluation of the query over the most likely set of trees.

\section{EFFICIENT QUERY PROCESSING}

Since node labels are unique in a tree, and a tree only has one root node, our goal will be to evaluate the root hub and the hubs containing node labels as early as possible. Since node numbers are also unique throughout the database, once a hub is chosen as a candidate for evaluation, its children also become bound uniquely. Therefore, it does not offer any computational advantage evaluating a subgoal $s_{i}$ with a few bound variables ahead of a subgoal $s_{j}$ with all free variables if $s_{j}$ will eventually be successful. But, if $s_{i}$ must fail (due to the bound variables), we want it to fail sooner so that we can stop processing the rest of the subgoals and save substantially, i.e., processing hubmatch ${ }^{f f}(\mathrm{~T} 1, \mathrm{~N} 6$, int, 2, _, [child (T1,N11, leaf,_,_), child(T1,10,int,_,_)] ) and

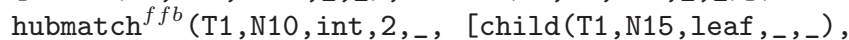
child $\left(\mathrm{T} 1, \mathrm{~N} 16\right.$, leaf $\left.\left.\left._{-}, l_{6}\right)\right]\right)$ in this order in $q_{4}$ after hubmatch $^{f b f}\left(\mathrm{~T} 1, \mathrm{~N} 21\right.$, int, 2, , [child(T1, N5, leaf , $\left.e_{1}, l_{1}\right)$, $\operatorname{child}\left(\mathrm{T} 1, \mathrm{~N} 6\right.$, int,,$\left.\left.{ }_{-}\right)\right]$), or vice-versa. While a comprehensive and exhaustive cost estimation is computationally prohibitive, based on these observations, we use the following simple heuristic method to order the query subgoals with a fail sooner strategy:

- Add target as the first subgoal to any query if $|\mathcal{T}|>0$.

- Order hubmatch and hedge subgoals in decreasing order of selectivity preferring the hedge subgoals.

- Add anc subgoals in decreasing order of number of bound variables.

- Finally, add Ica subgoals in decreasing order of number of bound variables.

In the above ordering strategy, we refine the subgoal order by prioritizing subgoals sharing higher number of variables through sideways information passing [17] over the ones sharing less, and also preferring EDB predicates having higher hub density, when all other parameters are comparable. For example, preferring hubmatch ${ }^{f f f}\left(\mathrm{~T} 1, \mathrm{~N} 1\right.$, root $, 3, \ldots_{\mathbf{H}^{-}}$, [child(T1,N2, int,_,_), child(T1,N3, leaf, , , $)$,

${ }^{7}$ Higher the selectivity, lesser are the number of instances and thus less cost for evaluation. 


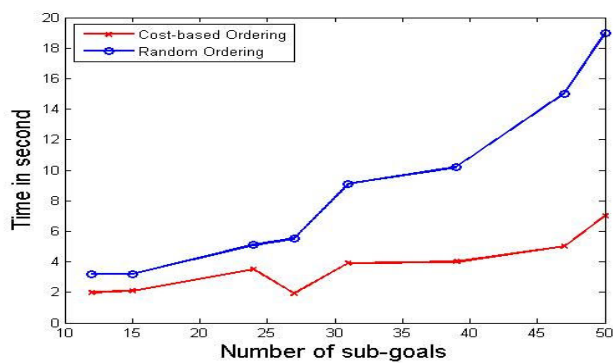

(a) EDB performance.

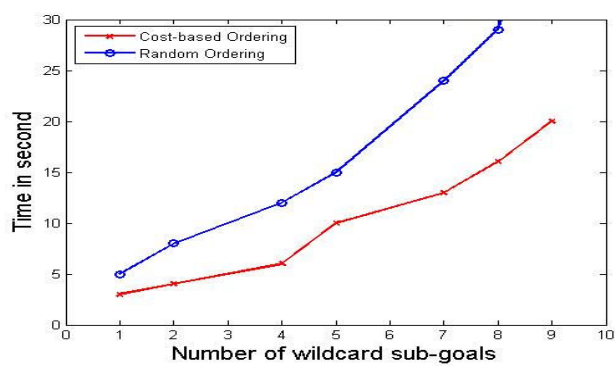

(b) Wildcard performance.

Figure 8: Preliminary performance results.

child (T1, N4, int, , , ) ]) over hubmatch ${ }^{f f}(\mathrm{~T} 1, \mathrm{~N} 6$, int , 2, , [child(T1,N11, leaf,_,_), child(T1,10,int,_,_)]). The strategy of subgoal ordering based on hub selectivity above yields substantial performance improvements as shown in figure 8 as opposed to a naive random subgoal ordering based on hub connectivity. In these two charts, we have plotted the query response times for several PhyQL queries over the TreeBASE database content. The figure in 8(a) shows performance improvement for EDB predicate ordering based on selectivity estimates, while figure 8(b) shows performance degradation with the increase in the number of wildcards in the queries. Note that queries in figure $8(\mathrm{~b})$ were also ordered for optimization based on cost-estimation for EDB predicates, and the number of wildcards that were mostly simple and shallow were selected randomly. However, to understand the true behavior of the wildcards, a more focused, objective and scientific experimentation is necessary in which the other EDB predicate profiles are kept constant, which was not the case in this preliminary experimentation. It was designed to observe only the gross performance profile and to understand the overall behavior.

\section{PHYLOBASE SYSTEM IMPLEMENTA- TION}

PhyloBase prototype was implemented using Java 1.6. Our implementation is based on XQJ Java API for XML documents supporting XQuery 1.0, which allows switching XQuery engines such as Saxon, BaseX, and eXist-DB as needed. We have, however, selected stable eXist-DB 2.2 for its superior performance and suitability in our current setting. Our experiments were run on a virtual computer equipped with a four-threaded Intel Xeon $3.00 \mathrm{GHz} \mathrm{CPU}$ and 16 GB RAM, running on Windows Server 2008 (64bits). The Prolog reasoner was implemented using a sta- ble version of SWI-Prolog 7.2.3 for Windows. That meant translating XML representation of PhyQL hubs into Prolog predicates and vice-versa. For this purpose, we have implemented a wrapper for the transformation in both directions. Since eXist-DB supports several indexing options for stored documents, in our current implementation, we have utilized its native indexing schemes for accessing the hubs instead of the ones we have proposed.

Regardless, the performance was significantly better than no indexing at all as shown in figure 8. Our expectation is that a judicious and custom implementation of our indexing schemes will help streamline the subgoal ordering strategy with accurate cost estimation directly from its native storage structure. A custom implementation of the proposed indices will also help further speed up the processing based on the cost estimation discussed in section 5.2 by partitioning the estimates per phylogeny at run time by judiciously selecting access strategies. For the current prototype, however, we have gathered the meta-data as an off line process scanning the eXist-DB storage and saving them in memory to aid cost estimation for the purpose of subgoal ordering.

\section{SUMMARY}

Query languages for phylogenies in particular, and for tree or graph databases in general, are limited. The major tree query languages closely follow the syntax and semantics of XQuery and XPath class of languages which we regard as overly procedural, and thus are not suitable for end users who are not programming savvy. Some have advocated using Neo4j's Cypher query language [14] for querying graphs and trees. But a closer look reveals that its strength comes at the expense of user friendliness and declarativity. Some studies (e.g., [39]) have also found that its performance is significantly poorer than other declarative graph query languages such as GraphQL [12]. Phylogeny and tree specific languages such as Crimson, TreeSearch, MXS [26] and Tregex [24] also do not support the much needed flexibility, declarativity and wildcard queries. Although the power and usefulness of declarative query languages for biological databases has been well-known [34, 35, 51], their rightful adoption has not kept pace.

In this paper, we have presented a query processing engine for an intuitive, flexible and declarative phylogeny query language called PhyQL introduced recently in [18], that is also visual. Its implementation and query processing rely on a deductive reasoner and thus allow significant query optimization opportunities, some of which have been discussed in this paper. While the eXist-DB based persistent storage and its native indexing scheme did not allow custom index implementation, opportunities for serious query optimization remain. In particular, we would like to adopt an improved list intersection procedure in the direction of [53] to expedite the target tree set identification using our indices. We have, however, shown that PhyQL still does significantly better with its subgoal ordering strategy, and it can do even better if we adopt a tree at a time processing strategy and use the proposed indices on the partitioned tree spaces logically partitioning the indices per phylogeny. Since our reasoner processes one tree at a time, we are then able to search optimally over a smaller space and fail sooner, if we must.

Augmenting eXist-DB with our custom indexing schemes, and developing a partitioned EDB search scheme that we 
skipped in the current release remains our future goal. Our hope is to compare PhyQL with some of the better known systems and popular tree query languages such as Cypher, GraphQL and VisualTregex, and XQuery in the axes of functionality, flexibility of querying and efficiency. We also would like to leverage the recent development in graph matching algorithms such as subgraph isomorph matching, and reachability to study if languages such as PhyQL can be efficiently implemented by judiciously mapping it to such algorithms and to achieve improved performance compared to a Datalog like reasoner based implementation.

\section{REFERENCES}

[1] N. S. Alghamdi, J. W. Rahayu, and E. Pardede. Semantic-based structural and content indexing for the efficient retrieval of queries over large XML data repositories. Future Generation Comp. Syst., $37: 212-231,2014$.

[2] B. Alix, D. A. Boubacar, and M. Vladimir. T-REX: a web server for inferring, validating and visualizing phylogenetic trees and networks. NAR, 2012.

[3] S. Amin, R. L. Finley, Jr., and H. M. Jamil. Top-k similar graph matching using TraM in biological networks. IEEE/ACM TCBB, 9(6):1790-1804, 2012.

[4] D. Bogdanowicz and K. Giaro. On a matching distance between rooted phylogenetic trees. Applied Mathematics and Computer Science, 23(3):669-684, 2013.

[5] J. G. Burleigh, M. S. Bansal, A. Wehe, and O. Eulenstein. Locating multiple gene duplications through reconciled trees. In International conference on Research in computational molecular biology, pages 273-284, 2008.

[6] J. Chai, H. Su, M. Wen, X. Cai, N. Wu, and C. Zhang. Resource-efficient utilization of $\mathrm{cpu} / \mathrm{gpu}$-based heterogeneous supercomputers for bayesian phylogenetic inference. The Journal of Supercomputing, 66(1):364-380, 2013.

[7] D. Chen, G. J. Burleigh, M. S. Bansal, and D. Fernandez-Baca. PhyloFinder: an intelligent search engine for phylogenetic tree databases. $B M C$ Evolutionary Biology, 8:90+, March 2008.

[8] B. Chisham, B. Wright, T. Le, T. Son, and E. Pontelli. CDAO-Store: Ontology-driven data integration for phylogenetic analysis. $B M C$ Bioinformatics, 12(1):98, 2011.

[9] J. Dutheil and N. Galtier. BAOBAB: a Java editor for large phylogenetic trees. Bioinformatics, 18(6):892-893, 2002.

[10] J. Felsenstein. The number of evolutionary trees. Systematic Zoology, 27(1):27+, Mar. 1978.

[11] P. A. Goloboff, S. A. Catalano, J. Marcos Mirande, C. A. Szumik, J. Salvador Arias, M. Källersjö, and J. S. Farris. Phylogenetic analysis of 73060 taxa corroborates major eukaryotic groups. Cladistics, 25(3):211-230, June 2009.

[12] H. He and A. Singh. Graphs-at-a-time: query language and access methods for graph databases. In SIGMOD, pages 405-418, 2008.

[13] S. Höhna, M. R. May, and B. R. Moore. TESS: an R package for efficiently simulating phylogenetic trees and performing bayesian inference of lineage diversification rates. Bioinformatics, 32(5):789-791, 2016.

[14] F. Holzschuher and R. Peinl. Performance of graph query languages: comparison of Cypher, Gremlin and native access in Neo4j. In EDBT/ICDT Workshops, pages 195-204, 2013.

[15] J. Huerta-Cepas, J. Dopazo, and T. Gabaldon. ETE: a python environment for tree exploration. $B M C$ Bioinformatics, 11(1):24, 2010.

[16] Y. Hulovatyy, H. Chen, and T. Milenkovic. Exploring the structure and function of temporal networks with dynamic graphlets. Bioinformatics, 31(12):171-180, 2015.

[17] Z. G. Ives and N. E. Taylor. Sideways information passing for push-style query processing. In ICDE, April 7-12, Cancún, México, pages 774-783, 2008.

[18] H. M. Jamil. A visual interface for querying heterogeneous phylogenetic databases. IEEE/ACM TCBB, 2016. DOI: 10.1109/TCBB.2016.2520943.

[19] G. Jin, L. Nakhleh, S. Snir, and T. Tuller. Efficient parsimony-based methods for phylogenetic network reconstruction. Bioinformatics, 23(2):123-128, 2007.

[20] J. Kohl, I. Paulsen, T. Laubach, A. Radtke, and A. von Haeseler. HvrBase++: a phylogenetic database for primate species. Nucleic Acids Res, 34(Database Issue):D700-D704, January 2005.

[21] B. R. Larget, S. K. Kotha, C. N. Dewey, and C. Ané. BUCKy: Gene tree / species tree reconciliation with bayesian concordance analysis. Bioinformatics, 2010.

[22] U. Leser. A query language for biological networks. Bioinformatics, 21(suppl 2):ii33-ii39, 2005.

[23] I. Letunic and P. Bork. Interactive Tree Of Life v2: online annotation and display of phylogenetic trees made easy. Nucleic Acids Research, 39(suppl 2):W475-W478, 2011.

[24] B. Levy and G. Andrew. Tregex and Tsurgeon: tools for querying and manipulating tree data structures. In Proceedings of the 5th international conference on Language Resources and Evaluation, pages 2231-2234, 2006.

[25] H. Li, A. Coghlan, J. Ruan, L. J. Coin, J. K. Hériché, L. Osmotherly, R. Li, T. Liu, Z. Zhang, L. Bolund, G. K. Wong, W. Zheng, P. Dehal, J. Wang, and R. Durbin. TreeFam: a curated database of phylogenetic trees of animal gene families. NAR, 34(Database issue):D572-80, January 2006.

[26] B. Ludäscher, I. Altintas, and A. Gupta. Time to leave the trees: From syntactic to conceptual querying of XML. In XML-Based Data Management and Multimedia Engineering - EDBT 2002 Workshops, Czech Republic, March 24-28, Revised Papers, pages 148-168, 2002.

[27] D. R. Maddison and K.-S. Schulz. The Tree of Life Web Project. http://tolweb.org, 2007.

[28] S. Marthey, G. Aguileta, F. Rodolphe, A. Gendrault, T. Giraud, E. Fournier, M. Lopez-Villavicencio, A. Gautier, M.-H. H. Lebrun, and H. Chiapello. FUNYBASE: a FUNgal phYlogenomic dataBASE. BMC bioinformatics, 9:456+, October 2008.

[29] C. Mathis, T. Härder, K. Schmidt, and S. Bächle. XML indexing and storage: fulfilling the wish list. 
Computer Science - RED, 30(1):51-68, 2015.

[30] L. Nakhleh, D. Miranker, F. Barbancon, W. H. Piel, and M. Donoghue. Requirements of phylogenetic databases. In IEEE BIBE, page 141, 2003.

[31] R. D. Natale, A. Ferro, R. Giugno, M. Mongiovì, A. Pulvirenti, and D. Shasha. SING: Subgraph search in non-homogeneous graphs. BMC Bioinformatics, 11:96, 2010.

[32] C. Ortutay, M. Siermala, and M. Vihinen. ImmTree: Database of evolutionary relationships of genes and proteins in the human immune system. Immunome Research, 3(4), March 2007.

[33] R. Page. Towards a Taxonomically Intelligent Phylogenetic Database. Nature Precedings, (713), September 2007.

[34] C. D. Page, Jr. The role of declarative languages in mining biological databases. In $P A D L$, page 1, 2003.

[35] J. M. Patel. The role of declarative querying in bioinformatics. OMICS, 7(1):89-92, 2003.

[36] M. Peterson and M. Colosimo. TreeViewJ: An application for viewing and analyzing phylogenetic trees. Source Code for Biology and Medicine, 2(1):7, 2007.

[37] S. Podell, T. Gaasterland, and E. E. Allen. A database of phylogenetically atypical genes in archaeal and bacterial genomes, identified using the DarkHorse algorithm. BMC bioinformatics, 9(1):419+, October 2008.

[38] V. Ranwez, N. Clairon, F. Delsuc, S. Pourali, N. Auberval, S. Diser, and V. Berry. PhyloExplorer: a web server to validate, explore and query phylogenetic trees. BMC Evolutionary Biology, 9(1):108+, May 2009.

[39] C. R. Rivero and H. M. Jamil. On isomorphic matching of large disk-resident graphs using an xquery engine. In IEEE ICDE Workshops, Chicago, IL, USA, March 31 - April 4, pages 20-27, 2014.

[40] C. R. Rivero and H. M. Jamil. Efficient and scalable labeled subgraph matching using SGMatch. Knowledge and Information Systems, 2016. To appear.

[41] V. Settepani, J. Bechsgaard, and T. Bilde. Phylogenetic analysis suggests that sociality is associated with reduced effectiveness of selection. Ecology and Evolution, 6(2):301-305, 2016.

[42] H. Shan, K. G. Herbert, W. H. Piel, D. Shasha, and J. T.-L. Wang. A structure-based search engine for phylogenetic databases. In SSDBM, pages 7-10, 2002.

[43] S. A. Smith, J. M. Beaulieu, A. Stamatakis, and M. J. Donoghue. Understanding angiosperm diversification using small and large phylogenetic trees. American Journal of Botany, 98(3):404-414, Mar. 2011.

[44] S. A. Smith, J. W. Brown, and C. E. Hinchliff. Analyzing and synthesizing phylogenies using tree alignment graphs. PLoS Comput Biololgy, 9(9):e1003223+, Sept. 2013.

[45] A. Stamatakis. Phylogenetics: Applications, software and challenges. Cancer Genomics and Proteomics, 2(5):301-305, 2005.

[46] H. H. Stedman, B. W. Kozyak, A. Nelson, D. M. Thesier, L. T. Su, D. W. Low, C. R. Bridges, J. B. Shrager, N. Minugh-Purvis, and M. A. Mitchell.
Myosin gene mutation correlates with anatomical changes in the human lineage. Nature, 428(6981):415-418, Mar. 2004.

[47] S. Sujatha, S. Balaji, and N. Srinivasan. PALI: a database of alignments and phylogeny of homologous protein structures. Bioinformatics, 17(4):375-376, April 2001.

[48] J. Sukumaran and M. T. Holder. DendroPy: a python library for phylogenetic computing. Bioinformatics, 26(12):1569-1571, June 2010.

[49] Z. Sun, H. Wang, H. Wang, B. Shao, and J. Li. Efficient subgraph matching on billion node graphs. PVLDB, 5(9):788-799, 2012.

[50] E. Talevich, B. Invergo, P. Cock, and B. Chapman. Bio.phylo: A unified toolkit for processing, analyzing and visualizing phylogenetic trees in biopython. $B M C$ Bioinformatics, 13(1):209, 2012.

[51] S. Tata, J. M. Patel, J. S. Friedman, and A. Swaroop. Declarative querying for biological sequences. In ICDE, page 87, 2006.

[52] V. Tropashko. Nested intervals tree encoding in sql. SIGMOD Rec., 34(2):47-52, 2005.

[53] D. Tsirogiannis, S. Guha, and N. Koudas. Improving the performance of list intersection. PVLDB, 2(1):838-849, 2009.

[54] V. Vesper. Lets do Dewey. http://www.mtsu.edu/ vvesper/dewey2.htm.

[55] R. A. Vos, H. Lapp, W. H. Piel, and V. Tannen. TreeBASE2: Rise of the Machines. Nature Precedings, (713), 2010.

[56] J. Wang, M. Guo, X. Liu, Y. Liu, C. Wang, L. Xing, and K. Che. Lnetwork: an efficient and effective method for constructing phylogenetic networks. Bioinformatics, 29(18):2269-2276, 2013.

[57] P. Wang, J. Tao, J. Zhao, and X. Guan. Moss: A scalable tool for efficiently sampling and counting 4and 5-node graphlets. CoRR, abs/1509.08089, 2015.

[58] Y.-C. Wu, M. D. Rasmussen, M. S. Bansal, and M. Kellis. TreeFix: Statistically informed gene tree error correction using species trees. Systematic Biology, 2012.

[59] B. Yelbay, S. I. Birbil, K. Bülbül, and H. M. Jamil. Approximating the minimum hub cover problem on planar graphs. Optimization Letters, 10(1):33-45, 2016.

[60] H. Zhang, S. Gao, M. J. Lercher, S. Hu, and W.-H. Chen. EvolView, an online tool for visualizing, annotating and managing phylogenetic trees. Nucleic Acids Research, 2012.

[61] S. Zhang, J. Yang, and W. Jin. SAPPER: Subgraph indexing and approximate matching in large graphs. PVLDB, 3(1):1185-1194, 2010.

[62] Y. Zheng, S. Fisher, S. Cohen, S. Guo, J. Kim, and S. B. Davidson. Crimson: a data management system to support evaluating phylogenetic tree reconstruction algorithms. In $V L D B$, pages 1231-1234, 2006.

[63] Y. Zhu, L. Qin, J. X. Yu, and H. Cheng. Finding top-k similar graphs in graph databases. In EDBT, pages 456-467, 2012. 